\title{
Effects of S-1 combined with palliative care on immune function and quality of life of patients with advanced stomach cancer
}

\author{
YING WANG and GUOZHONG LI \\ Department of Hematology, Tianjin Fifth Central Hospital, Tianjin 300450, P.R. China
}

Received January 20, 2020; Accepted May 8, 2020

DOI: 10.3892/ol.2020.11720

\begin{abstract}
The present study aimed to investigate the effects of S-1 combined with palliative care (PC) on the immune function and quality of life (QOL) of patients with advanced stomach cancer (ASC). In this prospective study, 168 patients with ASC admitted to our hospital from September 2016 to March 2018 were enrolled as research objects. Seventy-seven cases were treated with S-1 alone (single drug group, SDG), while another 91 cases were treated with S-1 combined with PC (combined drug group, $\mathrm{CDG}$ ). The effects of the two therapeutic methods on the efficacy [overall response rate (ORR)], 1-year overall survival rate (OSR), safety, negative emotions, nutritional indices, QOL, and immune function indices of patients were analyzed. After treatment, ORR, OSR, levels of nutritional indices [albumin (ALB), prealbumin (PA), and transferrin (TF)], and QOL improvement rate in the CDG were significantly higher than those in the SDG $(\mathrm{P}<0.05)$. After treatment, compared with those in the SDG, patients in the CDG had a lower Self-Rating Anxiety Scale (SAS) score, Self-Rating Depression Scale (SDS) score, and number of adverse reactions $(\mathrm{P}<0.05)$, and significantly improved immune function indices $\left(\mathrm{CD}^{+}, \mathrm{CD}^{+}\right.$, and $\left.\mathrm{CD}^{+} / \mathrm{CD}^{+}\right)(\mathrm{P}<0.05) . \mathrm{S}-1$ combined with $\mathrm{PC}$ treatment was superior to $\mathrm{S}-1$ treatment alone in patients with ASC. The patients treated with the combination exhibited improved efficacy (a higher ORR), higher QOL, and improved immune function, and thus this treatment can be clinically popularized.
\end{abstract}

\section{Introduction}

As one of the most common malignant tumors in the world, stomach cancer (SC) is the third leading cause of cancer-related deaths although its incidence has been decreasing in the past few decades $(1,2)$. According to the latest statistical report, more

Correspondence to: Dr Guozhong Li, Department of Hematology, Tianjin Fifth Central Hospital, 41 Zhejiang Road, Binhai New Area, Tianjin 300450, P.R. China

E-mail: gpz66i@163.com

Key words: S-1, palliative care, advanced stomach cancer, immune function, quality of life than 1 million people newly developed the disease worldwide in 2018, and the number of patients who succumbed to this disease was close to 800,000 , thus the disease poses a serious threat to human life and health (3). At present, surgery is the only potential radical treatment for SC, however the disease is mostly in its advanced stage when diagnosed and as a result, most patients are ineligible for radical surgery (4). Currently, chemotherapy is the first choice for SC patients who cannot undergo surgery, but there is no standard chemotherapeutic scheme with a satisfactory prognosis for them (5). Therefore, a current research hotspot is the discovery of a therapeutic scheme with better efficacy and higher safety to improve the prognosis of SC.

As an anticancer drug composed of tegafur (FT), gimeracil (CDHP), and oteracil (Oxo), $\mathrm{S}-1$ is well tolerated in clinical practice. This drug was approved for the treatment of advanced non-small cell lung cancer (NSCLC) in Japan in 2004, and then for the treatment of various malignant tumors including SC $(6,7)$. Palliative care (PC) is a multidisciplinary therapeutic method, and aims to improve the quality of life (QOL) of patients with serious diseases and their families (8). Its core components include the assessment and treatment of physical and psychological symptoms, the identification and support of mental pain, and the expert communication to set nursing goals and to assist in complex medical decisions and nursing coordination (9).

The application of S-1 combined with PC to patients with advanced stomach cancer (ASC) has been rarely explored. Therefore, the effects of the combination on the efficacy (ORR), survival rate, safety, negative emotions, QOL, and immune function of patients were observed in the present study, to provide more effective data for the clinical application of the therapeutic method.

\section{Materials and methods}

General information. This prospective study was approved by the Medical Ethics Committee of Tianjin Fifth Central Hospital (Tianjin, China). The research subjects included 168 patients (106 males and 62 females) with ASC treated at Tianjin Fifth Central Hospital from September 2016 to March 2018. Inclusion criteria for the patients were as follows: Patients aged $>18$ years; patients confirmed with SC by pathological examination; patients who had not taken drugs for the digestive tract system and anticancer drugs in the past month; 
patients in stages III and IV according to the TNM staging diagnostic criteria issued by American Joint Committee on Cancer (AJCC) in 2017 (10); patients who signed the informed consent form. Exclusion criteria for the patients were as follows: Patients with other malignant tumors except SC; patients with poor compliance; patients with expected survival time >3 months; patients who did not complete follow-up; patients with contraindications to the drugs used in this study; patients with incomplete clinical data.

Therapeutic methods. Patients in the single drug group (SDG; $n=77$ ) were orally administrated with S-1 (Taiho Pharmaceutical Co., Ltd.) twice a day, 40-60 mg each time according to their body surface area. Medication for 4 consecutive weeks was considered as one course of treatment, and the patients were treated for 2 courses. The second course started at 2 weeks after the first course. Those in the combined drug group (CDG; $n=91$ ) were treated as in the SDG group but were additionally treated with PC, which mainly included humanistic care, nutritional support, the prevention of toxic and side effects, and pain treatment. Following the treatment, the patients in both groups were followed-up by telephone and out-patient re-examinations to record their 1-year overall survival rate (OSR). The follow-up was conducted once a month and for a total of 12 months.

Outcome measures. The following day after the end of treatment, the clinical efficacy of the treatment on patients was assessed based on version 1.1 Response Evaluation Criteria in Solid Tumors (RECIST1.1) (11). The efficacy was classified into complete remission (CR), partial remission (PR), stable disease (SD), and progressive disease (PD). The overall response rate (ORR) was calculated as follows: $\mathrm{ORR}=(\mathrm{CR}+\mathrm{PR}$ cases $) /$ total number of cases $\times 100 \%$.

The occurrence of major adverse reactions during the treatment was observed, including loss of appetite, nausea and vomiting, leukopenia, diarrhea, insomnia, and bone marrow depression (BMD).

The Self-Rating Anxiety Scale (SAS) (12) and the Self-Rating Depression Scale (SDS) (13) were respectively used to score the anxiety and depression of the patients one day before and after treatment. Each scale had a total score of 100 points. A high score indicated serious anxiety and depression.

Serum samples were collected from the patients one day before and after treatment, in which levels of nutritional indices [albumin (ALB), prealbumin (PA), and transferrin (TF)] were measured using a Cobas C312 fully automatic biochemical analyzer (Roche Diagnostics).

At 15 days after treatment, the QOL of patients was evaluated according to Karnofsky Performance Scale (KPS) score (14). Improved indicated that the score was increased by $>10$ points. Stable indicated that the score was reduced or increased by $\leq 10$ points. Worsened indicated that the score was reduced by $>10$ points. The total improvement rate was calculated as follows: Total improvement rate=(improved + stable cases)/total number of cases $\times 100 \%$.

Serum samples were collected from the patients one day before and after treatment, in which peripheral blood $\mathrm{T}$ lymphocyte subsets were assessed using a FACSCalibur flow cytometer (BD Biosciences). Anticoagulated whole blood
(100 $\mu \mathrm{l})$ was respectively added with CD4-PE and CD8-PE antibodies (cat. nos. FAB3791P and FAB1509P, respectively; R\&D Systems, Inc.) (20 $\mu 1$ each), mixed well, and then allowed to stand at room temperature for $15 \mathrm{~min}$. Next, hemolysin (R\&D Systems, Inc.) (370 $\mu \mathrm{l})$ was added to the mixture, mixed well, and then allowed to stand at room temperature for $15 \mathrm{~min}$. Peripheral blood T lymphocyte subsets were detected on the flow cytometer.

Statistical analysis. In the present study, SPSS 21.0 (IBM Corp.) was used for statistical analysis. GraphPad Prism 7 (GraphPad Software, Inc.) was used to plot figures. Count data such as general information were expressed by $[\mathrm{n}(\%)]$, and the comparison of rate between groups was conducted by chi-square test. Measurement data such as SAS and SDS scores were expressed by (mean $\pm \mathrm{SD}$ ), and their comparison between groups was conducted by independent samples t-test, while the comparison within groups before and after treatment was conducted by paired t-test. Kaplan-Meier method was used to plot curves of the 1-year OSR after the follow-up. Log-rank test was used to analyze the difference in the survival between the two groups. $\mathrm{P}<0.05$ indicated a statistically significant difference.

\section{Results}

Comparison of general information. There were no significant differences between the SDG and the CDG in characteristics including sex, age, body weight, educational level, food preference, place of residence, exercise habits, marital status, history of smoking, history of drinking, TNM staging, and pathological types $(\mathrm{P}>0.05$; Table I).

Comparison of clinical efficacy. No patient died during the treatment. After treatment, there were 22 cases $(28.57 \%)$ of CR, 35 cases $(45.46 \%)$ of PR, 12 cases $(15.58 \%)$ of SD, and 8 cases $(10.39 \%)$ of PD in the SDG, with an ORR of $74.03 \%$. There were 32 cases $(35.16 \%)$ of CR, 48 cases $(52.75 \%)$ of PR, 6 cases $(6.59 \%)$ of SD, and 5 cases $(5.50 \%)$ of PD in the CDG, with an ORR of $87.91 \%$. After treatment, the ORR in the CDG was significantly higher than that in the SDG $(\mathrm{P}<0.05$; Table II).

Comparison of occurrence of adverse reactions. During the treatment, patients in the CDG and SDG had no drug allergy but good tolerance. Their major adverse reactions could be cured by clinical symptomatic treatment. There was no significant difference between the two groups in the occurrence of loss of appetite, insomnia, and BMD ( $P>0.05)$. The occurrence of nausea and vomiting, leukopenia, and diarrhea in the CDG was significantly lower than that in the SDG, and the total number of adverse reactions was also significantly lower in the CDG $(\mathrm{P}<0.05$; Table III).

Comparison of SAS and SDS scores. Before treatment, there were no significant differences between the SDG and CDG in SAS and SDS scores $(\mathrm{P}>0.05)$. After treatment, the two scores significantly decreased in the two groups, and the scores in the CDG were significantly lower than those in the SDG $(\mathrm{P}<0.05$; Table IV). 
Table I. Comparison of general information (mean \pm SD).

\begin{tabular}{|c|c|c|c|c|}
\hline Groups & SDG $(N=77)$ n $(\%)$ & $\mathrm{CDG}(\mathrm{N}=91) \mathrm{n}(\%)$ & $\chi^{2} / \mathrm{F}$-value & P-value \\
\hline Sex & & & 0.601 & 0.438 \\
\hline Male & $51(66.23)$ & $55(60.44)$ & & \\
\hline Female & $26(33.77)$ & $36(39.56)$ & & \\
\hline Age (years) & $56.24 \pm 8.67$ & $58.45 \pm 10.11$ & 1.506 & 0.134 \\
\hline BMI $\left(\mathrm{kg} / \mathrm{m}^{2}\right)$ & $23.13 \pm 1.89$ & $23.45 \pm 2.12$ & 1.024 & 0.307 \\
\hline Educational level & & & 0.881 & 0.348 \\
\hline$<$ Senior high school & $36(46.75)$ & $36(39.56)$ & & \\
\hline$\geq$ Senior high school & $41(53.25)$ & $55(60.44)$ & & \\
\hline Food preference & & & 1.062 & 0.303 \\
\hline Bland & $50(64.94)$ & $52(57.14)$ & & \\
\hline Greasy & $27(35.06)$ & $39(42.86)$ & & \\
\hline Place of residence & & & 2.907 & 0.088 \\
\hline City & $49(63.64)$ & $46(50.55)$ & & \\
\hline Countryside & $28(36.36)$ & $45(49.45)$ & & \\
\hline Exercise habits & & & 0.362 & 0.547 \\
\hline Yes & $39(50.65)$ & $33(36.26)$ & & \\
\hline No & $38(49.35)$ & $58(63.74)$ & & \\
\hline Marital status & & & 2.382 & 0.304 \\
\hline Married & $62(80.52)$ & $64(70.33)$ & & \\
\hline Unmarried & $6(7.79)$ & $12(13.19)$ & & \\
\hline Divorced & $9(11.69)$ & $15(16.48)$ & & \\
\hline History of smoking & & & 1.338 & 0.247 \\
\hline Yes & $55(71.43)$ & $72(79.12)$ & & \\
\hline No & $22(28.57)$ & $19(20.88)$ & & \\
\hline History of drinking & & & 0.887 & 0.346 \\
\hline Yes & $35(45.45)$ & $46(50.55)$ & & \\
\hline No & $42(54.55)$ & $45(49.45)$ & & \\
\hline TNM staging & & & 0.794 & 0.373 \\
\hline III & $37(48.05)$ & $50(54.95)$ & & \\
\hline IV & $40(51.95)$ & $41(45.05)$ & & \\
\hline Pathological types & & & 0.869 & 0.648 \\
\hline Adenocarcinoma & $65(84.42)$ & $72(79.12)$ & & \\
\hline Squamous cell carcinoma & $7(9.09)$ & $10(10.99)$ & & \\
\hline Signet-ring cell carcinoma & $5(6.49)$ & $9(9.89)$ & & \\
\hline
\end{tabular}

SDG, single drug group; CDG, combined drug group.

Comparison of changes in nutritional indices. Before treatment, there were no significant differences between the SDG and CDG in levels of ALB, PA, and TF (P>0.05). After treatment, the levels in the two groups significantly increased, and the levels in the CDG were significantly higher than those in the SDG $(\mathrm{P}<0.05$; Table $\mathrm{V})$.

Comparison of QOL improvement. In the SDG, the QOL was improved in 27 cases $(35.06 \%)$, stable in 32 cases $(41.56 \%)$, and worsened in 18 cases $(23.38 \%)$, with an improvement rate of $76.62 \%$. In the CDG, the QOL was improved in 39 cases $(42.86 \%)$, stable in 43 cases $(47.25 \%)$, and worsened in 9 cases $(9.89 \%)$, with an improvement rate of $90.11 \%$. The total improvement rate of the QOL in the CDG was significantly higher than that in the SDG $(\mathrm{P}<0.05$; Table VI).

Comparison of 1-year OSR. After the follow-up, the 1-year OSR in the CDG (57.14\%) was significantly higher than that in the SDG $(38.96 \%)(\mathrm{P}<0.05$, log-rank test; Fig. 1$)$.

Comparison of immune function indices before and after treatment. Before treatment, there were no significant differences between the SDG and CDG in levels of serum CD4 ${ }^{+}$, $\mathrm{CD}^{+}$, and $\mathrm{CD}^{+} / \mathrm{CD}^{+}(\mathrm{P}>0.05)$. After treatment, the levels of $\mathrm{CD}^{+}$and $\mathrm{CD}^{+} / \mathrm{CD}^{+}$in the two groups significantly increased, and the levels in the CDG were significantly higher 
Table II. Comparison of clinical efficacy.

\begin{tabular}{|c|c|c|c|c|c|c|}
\hline Groups & $\mathrm{n}$ & CR n (\%) & PR n (\%) & SD n $(\%)$ & PD n $(\%)$ & ORR n $(\%)$ \\
\hline $\mathrm{SDG}$ & 77 & $22(28.57)$ & $35(45.46)$ & $12(15.58)$ & 8 (10.39) & $57(74.03)$ \\
\hline $\mathrm{CDG}$ & 91 & $32(35.16)$ & $48(52.75)$ & $6(6.59)$ & $5(5.50)$ & $80(87.91)$ \\
\hline$\chi^{2}$ test & - & - & - & - & - & 5.345 \\
\hline P-value & - & - & - & - & - & 0.021 \\
\hline
\end{tabular}

$\mathrm{CR}$, complete remission; $\mathrm{PR}$, partial remission; $\mathrm{SD}$, stable disease; PD, progressive disease; ORR, overall response rate; SDG, single drug group; CDG, combined drug group.

Table III. Comparison of occurrence of adverse reactions.

\begin{tabular}{lcrr}
\hline Groups & SDG $(\mathrm{N}=77) \mathrm{n}(\%)$ & CDG $(\mathrm{N}=91) \mathrm{n}(\%)$ & $\chi^{2}$ test \\
\hline Loss of appetite & $14(18.18)$ & $8(8.79)$ & 3.232 \\
Nausea and vomiting & $22(28.57)$ & $12(13.79)$ & 0.072 \\
Leukopenia & $12(15.58)$ & $5(5.49)$ & 0.013 \\
Diarrhea & $16(20.78)$ & $8(8.79)$ & 4.669 \\
Insomnia & $11(14.29)$ & $7(7.69)$ & 4.895 \\
BMD & $7(9.09)$ & $5(5.49)$ & 1.895 \\
Total number of adverse reactions & $39(50.65)$ & $29(31.87)$ & 0.031 \\
\hline
\end{tabular}

SDG, single drug group; CDG, combined drug group; BMD, bone marrow depression.

Table IV. Comparison of SAS and SDS scores (mean \pm SD, points).

\begin{tabular}{|c|c|c|c|c|}
\hline \multirow[b]{2}{*}{ Groups } & \multicolumn{2}{|c|}{ SAS } & \multicolumn{2}{|c|}{ SDS } \\
\hline & Before treatment & After treatment & Before treatment & After treatment \\
\hline $\operatorname{SDG}(n=77)$ & $86.56 \pm 10.54$ & $78.56 \pm 11.54^{\mathrm{a}}$ & $85.86 \pm 10.12$ & $76.51 \pm 12.52^{\mathrm{a}}$ \\
\hline $\operatorname{CDG}(\mathrm{n}=91)$ & $88.56 \pm 9.38$ & $69.56 \pm 8.54^{\mathrm{a}}$ & $87.56 \pm 9.68$ & $70.23 \pm 9.56^{\mathrm{a}}$ \\
\hline t-value & 1.301 & 5.798 & 1.111 & 3.682 \\
\hline P-value & 0.195 & $<0.001$ & 0.268 & $<0.001$ \\
\hline
\end{tabular}

ap $<0.05$, compared with the before treatment of the same group. SAS, Self-Rating Anxiety Scale; SDS, Self-Rating Depression Scale; SDG, single drug group; $\mathrm{CDG}$, combined drug group.

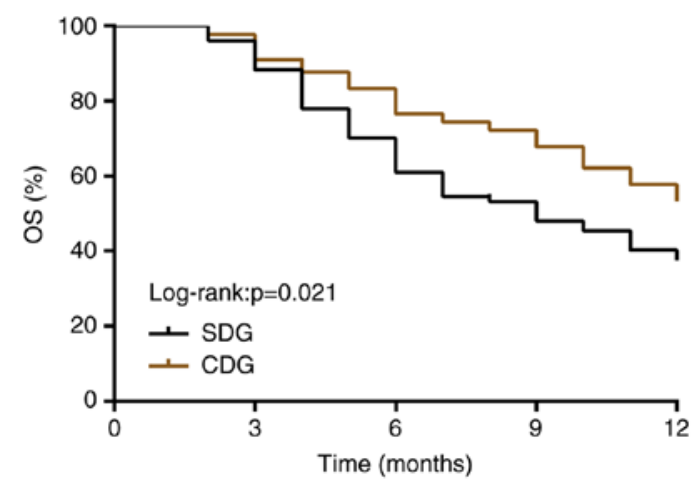

Figure 1. Comparison of 1-year OSR. The 1-year OSR in the CDG was significantly higher than that in the SDG. OSR, overall survival rate; $\mathrm{CDG}$, combined drug group; SDG, single drug group. than those in the SDG $(\mathrm{P}<0.05)$. However, the $\mathrm{CD}^{+}$level in the two groups significantly decreased, and the level in the CDG was significantly lower than that in the SDG $(\mathrm{P}<0.05$; Fig. 2).

\section{Discussion}

Changes in the life and dietary habits of people leads to a gradual increase in the incidence of digestive system diseases (15). In addition to a common malignant tumor of the digestive system worldwide and a major factor of cancer-related deaths (16), SC is also a genetically heterogeneous tumor with multiple causes, closely related to heredity, helicobacter pylori infection, diet, and lifestyle (17). ASC is characterized by high metastasis and high recurrence, which result in the worst clinical results of 
Table V. Comparison of changes in nutritional indices (mean $\pm \mathrm{SD})$.

\begin{tabular}{|c|c|c|c|c|c|c|}
\hline \multirow[b]{2}{*}{ Groups } & \multicolumn{2}{|c|}{$\operatorname{ALB}(\mathrm{g} / \mathrm{l})$} & \multicolumn{2}{|c|}{$\mathrm{PA}(\mathrm{mg} / \mathrm{l})$} & \multicolumn{2}{|c|}{$\mathrm{TF}(\mathrm{g} / \mathrm{l})$} \\
\hline & Before treatment & After treatment & Before treatment & After treatment & Before treatment & After treatment \\
\hline $\operatorname{SDG}(n=77)$ & $28.15 \pm 3.78$ & $33.34 \pm 4.21^{\mathrm{a}}$ & $125.14 \pm 18.61$ & $192.24 \pm 25.34^{\mathrm{a}}$ & $1.38 \pm 0.34$ & $1.72 \pm 0.33^{\mathrm{a}}$ \\
\hline CDG $(n=91)$ & $28.89 \pm 4.13$ & $36.67 \pm 4.45^{\mathrm{a}}$ & $127.34 \pm 20.43$ & $257.15 \pm 27.67^{\mathrm{a}}$ & $1.43 \pm 0.36$ & $2.27 \pm 0.41^{\mathrm{a}}$ \\
\hline t-value & 1.203 & 4.953 & 0.724 & 15.743 & 0.920 & 9.460 \\
\hline P-value & 0.231 & $<0.001$ & 0.470 & $<0.001$ & 0.359 & $<0.001$ \\
\hline
\end{tabular}

${ }^{\mathrm{a}} \mathrm{P}<0.05$, compared with the before treatment of the same group. ALB, albumin; PA, prealbumin; TF, transferrin; SDG, single drug group; CDG, combined drug group.

Table VI. Comparison of QOL improvement.

\begin{tabular}{lccccc}
\hline Groups & $\mathrm{N}$ & Improved n (\%) & Stable n (\%) & Worsened n (\%) & Total improvement rate n (\%) \\
\hline SDG & 77 & $27(35.06)$ & $32(41.56)$ & $18(23.38)$ & $59(76.62)$ \\
CDG & 91 & $39(42.86)$ & $43(47.25)$ & $9(9.89)$ & $82(90.11)$ \\
$\chi^{2}$ & - & - & - & - & 5.624 \\
P-value & - & - & - & - & 0.018 \\
\hline
\end{tabular}

QOL, quality of life; SDG, single drug group; CDG, combined drug group.
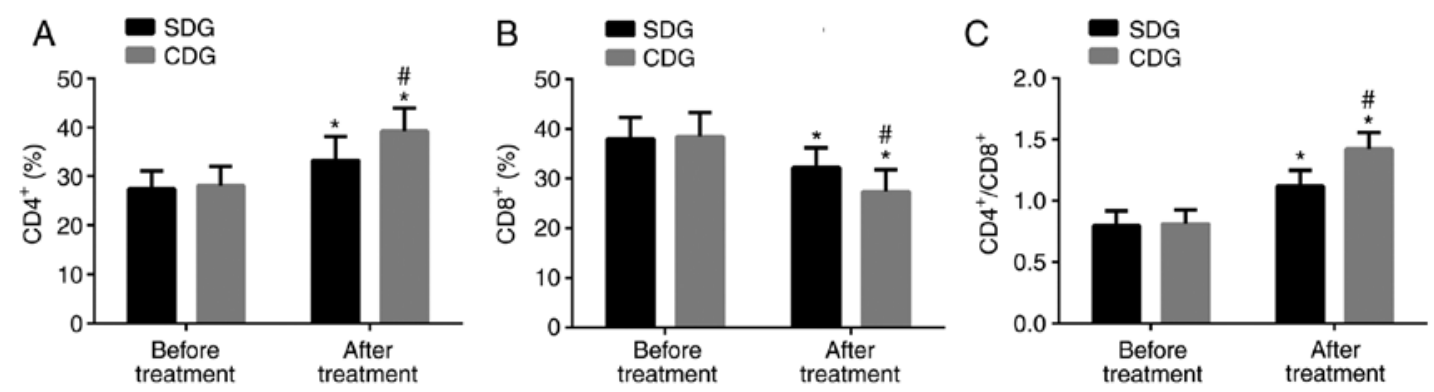

Figure 2. Changes in cellular immune indices. (A) After treatment, the $\mathrm{CD} 4^{+}$level in the two groups was significantly increased, and the level in the CDG was significantly higher than that in SDG. (B) After treatment, the CD8 ${ }^{+}$level in the two groups was significantly decreased, and the level in the CDG was significantly lower than that in the SDG. (C) After treatment, the $\mathrm{CD} 4^{+} / \mathrm{CD} 8^{+}$level in the two groups was significantly increased, and the level in the $\mathrm{CDG}$ was significantly higher than that in the SDG. ${ }^{~} \mathrm{P}<0.05$ compared with the before treatment of the same group. ${ }^{*} \mathrm{P}<0.05$ compared with the SDG. CDG, combined drug group; SDG, single drug group.

the disease among all solid organ tumors (18). Therefore, the therapeutic effect on ASC urgently requires improvement in clinical practice.

FT is a prodrug converted into 5-fluorouracil (5-Fu), whose catabolism can be delayed by CDHP and whose phosphorylation can be inhibited by Oxo (19). S-1 that is composed of the three drugs can prolong and maintain 5-Fu concentration and reduce its toxicity (19). This drug is common for the treatment of various tumor diseases including SC (20-22). PC, an interdisciplinary specialty, is dedicated to improving the QOL of critically ill patients and their families through symptom management, communication, and patient autonomy (23). Its combination with chemotherapy is commonly used to treat tumors, but there are currently few studies on S-1 combined with PC to treat ASC. In the study, the ORR and 1-year OSR in the CDG were significantly higher than those in the SDG, which demonstrated that S-1 combined with PC has a better efficacy and higher safety than S-1 alone in the treatment of patients with ASC. Improving the QOL is one of the basic goals of treatment, however cancer patients, especially those with advanced cancers, have a markedly decreased QOL owing to disease symptoms, pain, and negative emotions $(24,25)$. In addition, patients with ASC suffer from less food intake and poor absorption caused by the disease itself or the toxic and side effects of chemotherapeutic drugs, eventually resulting in the patients experiencing malnutrition which directly affects their QOL $(26,27)$. Therefore, apart from the treatment of the disease itself, all efforts should be made to appease the emotions of the patients and provide adequate nutritional support, thus improving the QOL more effectively. In the present study, compared with those in the SDG, patients in the CDG had a decreased number of adverse reactions, SAS 
score, and SDS score, but an increased QOL improvement rate and levels of nutritional indices (ALB, PA, and TF). This indicated that S-1 combined with PC had higher safety, and could improve the QOL of patients with ASC more significantly, because PC aims to improve the QOL of patients with serious diseases and their families through humanistic care, symptomatic treatment, as well as other means.

The immune system has a great effect on controlling and curing cancers, and the immune function of cancer patients is generally inhibited compared with that of healthy people (28). Accordingly, immunotherapy for tumors has been widely valued in the medical field, and thus it has a broad application prospect (29). Studies have revealed that nutritional support has a great effect on improving the immune function of the cancer patients $(30,31)$. T lymphocyte-mediated cellular immunity plays a pivotal role in the antitumor immune mechanism of the body, thus changes in Tlymphocyte subsets $\left(\mathrm{CD}^{+}\right.$and $\left.\mathrm{CD}^{+}\right)$are important markers reflecting immune dysfunction $(32,33)$. In the present study, post-treatment levels of $\mathrm{CD}^{+}$and $\mathrm{CD} 4^{+} / \mathrm{CD}^{+}$in the $\mathrm{CDG}$ were higher than those in the SDG, while the post-treatment $\mathrm{CD}^{+}$level was lower than that in the SDG, indicating that S-1 combined with PC has a better effect on relieving immunosuppression in patients with ASC. This may be due to the fact that nutritional support provided to the patients in the CDG provides various nutrients, and thereby improves their immune function.

In summary, $\mathrm{S}-1$ combined with $\mathrm{PC}$ is more effective than S-1 alone in the treatment of patients with ASC. The patients treated with the combination had improved efficacy (higher ORR), higher QOL, and better immune function, and thus this treatment can be clinically popularized. However, there are some shortcomings in the present study. Firstly, the number of the research subjects was small and the follow-up time was short, which may lead to bias in the K-M survival analysis. Secondly, the QOL of the family members of the patients and the disease-free survival time were not investigated. Additionally, the cost for these treatments was not recorded. Therefore, our aim is to improve these deficiencies in future studies.

\section{Acknowledgements}

Not applicable.

\section{Funding}

No funding was received.

\section{Availability of data and materials}

The datasets used and/or analyzed during the current study are available from the corresponding author on reasonable request.

\section{Authors' contributions}

YW wrote the manuscript, interpreted and analyzed the data. GL designed the study and performed the experiment. YW was responsible for the analysis and discussion of the data. Both authors read and approved the final manuscript.

\section{Ethics approval and consent to participate}

The study was approved by the Medical Ethics Committee of Tianjin Fifth Central Hospital (Tianjin, China). Patients who participated in this research, provided signed informed consent and had complete clinical data. Signed written informed consents were obtained from the patients and/or guardians.

\section{Patient consent for publication}

Not applicable.

\section{Competing interests}

The authors declare that they have no competing interests.

\section{References}

1. Zhang Y, Liu H, Li W, Yu J, Li J, Shen Z, Ye G, Qi X and Li G: CircRNA_100269 is downregulated in gastric cancer and suppresses tumor cell growth by targeting miR-630. Aging (Albany NY) 9: 1585-1594, 2017.

2. Sitarz R, Skierucha M, Mielko J, Offerhaus GJA, Maciejewski R and Polkowski WP: Gastric cancer: Epidemiology, prevention, classification, and treatment. Cancer Manag Res 10: 239-248, 2018,

3. Bray F, Ferlay J, Soerjomataram I, Siegel RL, Torre LA and Jemal A: Global cancer statistics 2018: GLOBOCAN estimates of incidence and mortality worldwide for 36 cancers in 185 countries. CA Cancer J Clin 68: 394-424, 2018.

4. Wagner AD, Syn NL, Moehler M, Grothe W, Yong WP, Tai BC, Ho J and Unverzagt S: Chemotherapy for advanced gastric cancer. Cochrane Database Syst Rev 8: CD004064, 2017.

5. Xu W, Yang Z and Lu N: Molecular targeted therapy for the treatment of gastric cancer. J Exp Clin Cancer Res 35: 1, 2016.

6. Li F, Ju Y, Guan Y and Zhao H: Tegafur gimeracil oteracil potassium capsule induced acute interstitial lung disease: A case report: Zhongguo Fei Ai Za Zhi 17: 53-56, 2014 (In Chinese).

7. Kobayakawa M and Kojima Y: Tegafur/gimeracil/oteracil (S-1) approved for the treatment of advanced gastric cancer in adults when given in combination with cisplatin: A review comparing it with other fluoropyrimidine-based therapies. Onco Targets Ther 4: 193-201, 2011 .

8. Zimmermann C, Swami N, Krzyzanowska M, Leighl N, Rydall A, Rodin G, Tannock I and Hannon B: Perceptions of palliative care among patients with advanced cancer and their caregivers. CMAJ 188: E217-E227, 2016.

9. Kelley AS and Morrison RS: Palliative care for the seriously ill. N Engl J Med 373: 747-755, 2015.

10. In H, Solsky I, Palis B, Langdon-Embry M, Ajani J and Sano T: Validation of the 8th edition of the AJCC TNM staging system for gastric cancer using the national cancer database. Ann Surg Oncol 24: 3683-3691, 2017.

11. Eisenhauer A and Verweij J: 11 New response evaluation criteria in solid tumors: RECIST GUIDELINE VERSION 1.1. Eur J Cancer Suppl 7: 5, 2009.

12. Chen ML, Liu J, Zhang J and Wu TJ: Investigation for the incidences of cognitive emotion regulation, quality of life, anxiety and depression in patients with chronic heart failure. Chin Circ J 32: 956-959, 2017.

13. Liu N, Liu S, Yu N, Peng Y, Wen Y, Tang J and Kong L: Correlations among psychological resilience, self-efficacy, and negative emotion in acute myocardial infarction patients after percutaneous coronary intervention. Front Psychiatry 9: 1, 2018.

14. Chang JY, Verma V, Li M, Zhang W, Komaki R, Lu C, Allen PK, Liao Z, Welsh J, Lin SH, et al: Proton beam radiotherapy and concurrent chemotherapy for unresectable stage III non-small cell lung cancer: Final results of a phase 2 study. JAMA Oncol 3: e172032, 2017.

15. Van Cutsem E, Sagaert X, Topal B, Haustermans K and Prenen H: Gastric cancer. Lancet 388: 2654-2664, 2016.

16. Plummer M, Franceschi S, Vignat J, Forman D and de Martel C: Global burden of gastric cancer attributable to helicobacter pylori. Int J Cancer 136: 487-490, 2015.

17. Cheng XJ, Lin JC and Tu SP: Etiology and prevention of gastric cancer. Gastrointest Tumors 3: 25-36, 2016. 
18. Liu Y, Feng Y, Gao Y and Hou R: Clinical benefits of combined chemotherapy with S-1, oxaliplatin, and docetaxel in advanced gastric cancer patients with palliative surgery. Onco Targets Ther 9: 1269-1273, 2016.

19. Lv X, Zhang L, Huang R and Song W: A clinical exploration of neoadjuvant chemotherapy with tegafur, gimeracil, and oteracil potassium capsules combined with oxaliplatin for advanced gastric cancer. Int J Clin Exp Med 8: 19030-19036, 2015.

20. Yeo W, Lam KO, Law LY, Lee CC, Chiang CL, Au KH, Mo FK, So TH, Lam KC, Ng WT and Li L: Adjuvant S-1 chemotherapy after curative resection of gastric cancer in Chinese patients: Assessment of treatment tolerability and associated risk factors. Hong Kong Med J 23: 54-62, 2017.

21. Otsuka H, Fujii T, Toh U, Iwakuma N, Takahashi R, Mishima M, Takenaka M, Kakuma T, Tanaka M and Shirouzu K: Phase II clinical trial of metronomic chemotherapy with combined irinotecan and tegafur-gimeracil-oteracil potassium in metastatic and recurrent breast cancer. Breast Cancer 22: 335-342, 2015.

22. Wang T, Zhang SF, Qiu MQ and Li QL: Efficacy and safety of S-1 (tegafur, gimeracil, and oteracil potassium) concurrent with 3-dimensional conformal radiotherapy for newly diagnosed squamous cell carcinoma of the lung in elderly patients. Cancer Radiother 20: 181-186, 2016.

23. May P, Normand C, Cassel JB, Del Fabbro E, Fine RL, Menz R, Morrison CA, Penrod JD, Robinson C and Morrison RS: Economics of palliative care for hospitalized adults with serious illness: A meta-analysis. JAMA Intern Med 178 : 820-829, 2018

24. Polanski J, Jankowska-Polanska B, Rosinczuk J, Chabowski M and Szymanska-Chabowska A: Quality of life of patients with lung cancer. Onco Targets Ther 9: 1023-1028, 2016.
25. Park SA, Chung SH and Lee Y: Factors influencing the quality of life of patients with advanced cancer. Appl Nurs Res 33: 108-112, 2017.

26. Bicakli DH, Ozveren A, Uslu R, Dalak RM, Cehreli R, Uyar M, Karabulut B and Akcicek F: The effect of chemotherapy on nutritional status and weakness in geriatric gastrointestinal system cancer patients. Nutrition 47: 39-42, 2018.

27. Cotogni P, De Carli L, Passera R, Amerio ML, Agnello E, Fadda M, Ossola M, Monge T, De Francesco A and Bozzetti F: Longitudinal study of quality of life in advanced cancer patients on home parenteral nutrition. Cancer Med 6: 1799-1806, 2017.

28. Garcia-Anguita A, Kakourou A and Tsilidis KK: Biomarkers of inflammation and immune function and risk of colorectal cancer. Curr Colorectal Cancer Rep 11: 250-258, 2015.

29. Li K, Zhang Q, Zhang Y, Yang J and Zheng J: T-cell-associated cellular immunotherapy for lung cancer. J Cancer Res Clin Oncol 141: 1249-1258, 2015.

30. Wang F, Hou MX, Wu XL, Bao LD and Dong PD: Impact of enteral nutrition on postoperative immune function and nutritional status. Genet Mol Res 14: 6065-6072, 2015.

31. Ding D, Feng Y, Song B, Gao S and Zhao J: Effects of preoperative and postoperative enteral nutrition on postoperative nutritional status and immune function of gastric cancer patients. Turk J Gastroenterol 26: 181-185, 2015.

32. Sun H, Song Y and Wang XY: Effects of different anesthetic methods on cellular immune and neuroendocrine functions in patients with hepatocellular carcinoma before and after surgery. J Clin Lab Anal 30: 1175-1182, 2016.

33. Ivanova EA and Orekhov AN: T helper lymphocyte subsets and plasticity in autoimmunity and cancer: An overview. Biomed Res Int 2015: 327470, 2015. 